\title{
The Treatment Effectiveness Evaluation for Slowing the Progression of Diabetic Nephropathy During Stage 4 Chronic Kidney Disease
}

\author{
Tianyu Yu $\cdot$ Shimin Jiang $\cdot$ Yue Yang · Jinying Fang $\cdot$ Guming Zou • \\ Hongmei Gao $\cdot$ Li Zhuo · Wenge Li (D)
}

Received: October 6, 2020 / Accepted: November 12, 2020 / Published online: November 29, 2020

(C) The Author(s) 2020

\begin{abstract}
Introduction: People with advanced diabetic nephropathy (DN) are at high risk for development of end-stage renal disease (ESRD) or death. Whether renin-angiotensin system inhibitors and some concomitant drugs could still continue to delay the onset of ESRD in the later stage of DN needs to be clarified. This study aimed to evaluate the relationship of the therapeutic methods as well as clinicopathologic variables with prognosis of patients with biopsy-proven DN during stage 4 of chronic kidney disease (CKD).
\end{abstract}

T. Yu · S. Jiang $(\bowtie) \cdot$ Y. Yang · G. Zou · H. Gao .

L. Zhuo · W. Li $(\bowtie)$

Department of Nephrology, China-Japan Friendship

Hospital, No. 2 East Yinghuayuan Street, Chaoyang

District, Beijing 100029, China

e-mail: minjiang101@163.com

W. Li

e-mail: wenge_lee2002@126.com

T. Yu $\cdot$ W. Li

Graduate School of Peking Union Medical College, Peking Union Medical College and Chinese Academy of Medical Sciences, Beijing 100730, China

J. Fang

Beijing University of Chinese Medicine, Beijing 100029, China
Methods: Forty-six DN patients who underwent renal biopsy in stage 4 CKD were enrolled from January 1, 2002, to December 31, 2019. Clinical data were abstracted retrospectively from the time of renal biopsy. Follow-up data were collected until April 1, 2020, or from the day of renal biopsy to either the occurrence of ESRD or death. The primary outcome was the composite of ESRD or death. Treatment effectiveness and the prognostic ability of clinicopathologic data were evaluated using multivariate Cox regression analyses.

Results: The median renal survival duration was 17.3 (95\% confidence interval, 7.4-27.3 months). Primary endpoint events occurred in 29 individuals (63.0\%) during follow-up, including 24 who reached ESRD and 5 who died before progression to ESRD. None of the clinicopathologic data, including pathologic cass of DN, were statistically independent prognostic factors for renal survival. Conventional therapies, such as use of renin-angiotensin system (RAS) inhibitors, a level of glycated hemoglobin (HbA1c) $<7 \%$, and blood pressure $<130 /$ $80 \mathrm{mmHg}$, were also not statistically different between the stable and progressive groups.

Conclusion: Specific therapies including targeting blood pressure $<130 / 80 \mathrm{mmHg}$, HbA1c concentration $<7 \%$, and use of RAS inhibitors could not effectively delay the onset of ESRD in the later stage of DN. Therefore, efforts to slow the progression of $\mathrm{DN}$ should focus on early diagnosis and treatment. 
Keywords: Angiotensin II type 1 receptor blocker; Diabetic nephropathy; Prognosis; Therapy

\section{Key Summary Points}

Why carry out this study?

Diabetic nephropathy (DN) continues to be the leading cause of end-stage renal disease (ESRD) worldwide, and the progression rates of different stages of DN differ.

Whether routine therapies are still effective in delaying the onset of ESRD in the later stage of DN needs to be further clarified.

\section{What was learned from the study?}

The median time for DN during stage 4 chronic kidney disease to progress to ESRD was 17.3 months.

Routine treatments, such as inhibition of RAS, glycemic control, blood pressure control, and use of Rheum officinale, could not effectively forestall the onset of ESRD.

The results of this study suggest that therapy to forestall the progression of DN should focus upon early intervention.

\section{DIGITAL FEATURES}

This article is published with digital features, including a summary slide, to facilitate understanding of the article. To view digital features for this article go to https://doi.org/10.6084/ m9.figshare.13221869.

\section{INTRODUCTION}

Diabetic nephropathy (DN) has become the leading cause of end-stage renal disease (ESRD) in most developed countries [1] and is predicted to be one of the top seven causes of death worldwide [2]. In the past 2 decades, the increasing prevalence of diabetes and premature mortality has placed a huge burden on society $[3,4]$. How to effectively slow the progression of DN is thereby an important medical and social issue in the treatment of DN.

Specific treatments of patients with DN, such as glycemic control, blood pressure control, and inhibition of the renin-angiotensin system (RAS), are the main methods for delaying the progression of DN [5]. Additional therapy, such as the Chinese patented medicine Rheum (CPM-Rheum), which offers a range of pharmacologic properties that may delay disease progression, was also often used in China to treat CKD $[6,7]$. The most widely used therapy for DN is therapy with a RAS-blocking medication because of its renoprotective effects [8]. However, to avoid increasing the risk of hyperkalemia, using a RAS-blocking agent for the first time in advanced DN, such as stage $4 \mathrm{CKD}$, is not recommended; for those with later stages of DN who have already been using a RAS-blocking medication, discontinuation is not necessary.

To investigate whether conventional therapy could continue to forestall the onset of ESRD in the later stage of DN, we therefore enrolled all patients with DN who underwent kidney biopsy during stage 4 CKD in our center. We collected clinicopathologic data, the history of RASblocking medication as well as additional therapy and focused on the level of expression of the angiotensin II type 1 receptor (AT1R). The objective of our study was to clarify the association of therapeutic methods as well as clinicopathologic variables with the prognosis of patients in the later stage of $\mathrm{DN}$.

\section{METHODS}

\section{Study Design and Participants}

This was a retrospective study. We enrolled patients with type 1 or type 2 diabetes and kidney disease who underwent a renal biopsy at the China-Japan Friendship Hospital from January 1, 2002, to December 31, 2019. The renal biopsy operators were clinicians with many 
years of relevant experience in our department. The patient inclusion criteria were: (1) biopsyproven $\mathrm{DN}$; (2) patients undergoing renal biopsy in stage CKD 4 (eGFR between 15 and $30 \mathrm{ml} / \mathrm{min} / 1.73 \mathrm{~m}^{2}$ ). The exclusion criteria were DN patients with other nephropathies, such as non-diabetic renal disease, who had a biopsy specimen with $<5$ total glomeruli and who were followed up in our hospital for $<4$ months (Fig. 1).

The protocol of this study was approved by the institutional ethics committee for human research of the China-Japan Friendship Hospital (2018-43-K32). All the procedures that included human participants adhered to the Declaration of Helsinki. All patients provided informed consent before undergoing renal biopsy.

\section{Parameters and Definitions}

The following clinical parameters were collected from the electronic medical system: sex, age, body mass index (BMI), duration of diabetes (from diagnosis of diabetes to renal biopsy, years), 24-h urinary protein excretion (UPE, $\mathrm{g} /$ day), serum creatinine $(\mu \mathrm{mol} / \mathrm{l})$, serum albumin ( $\mathrm{g} / \mathrm{l})$, fasting blood glucose (FBG, mmol/l), glycated hemoglobin (HbA1c, \%), hemoglobin $(\mathrm{Hb}, \mathrm{g} / \mathrm{l})$, cholesterol, triglyceride $(\mathrm{mmol} / \mathrm{l})$, potassium, phosphate, brain natriuretic peptide (BNP), parathyroid hormone, systolic blood pressure (SBP), diastolic blood pressure (DBP),

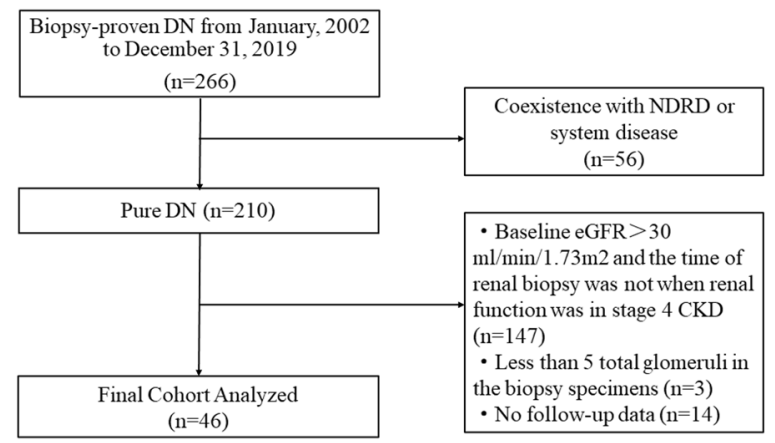

Fig. 1 Flowchart of study participants. $D N$ diabetic nephropathy, $N D R D$ non-diabetic renal disease, $e G F R$ estimated glomerular filtration rate. ${ }^{*} \mathrm{DN}$ patients with eGFR $<15 \mathrm{ml} / \mathrm{min} / 1.73 \mathrm{~m}^{2}$ were not included in this study whether the blood pressure target was reached, and history of cardiovascular events (CVEs). Meanwhile, we collected the history of using Rheum officinale. We calculated eGFR using the creatinine-based Chronic Kidney Disease Epidemiology Collaboration (CKD-EPI) equation [9].

Target blood pressure was defined as < $130 / 80 \mathrm{mmHg}$ [10]. HbA1c $<7 \%$ was defined as a reasonable level for adults [11]. The history of using angiotensin II type 1 receptor blocker (ARB) therapy was defined as continuing oral administration of ARB drugs before renal biopsy. Erythropoietin-stimulating agent (ESA) therapy was defined as the correction of anemia according to clinical needs. CVEs were defined as a history of diseases with a clear diagnosis, including acute coronary syndrome, heart failure, arrhythmia, revascularization events (percutaneous transluminal coronary angioplasty and coronary artery bypass grafting), and stroke.

\section{Endpoints and Follow-Up}

The primary endpoints were ESRD or death. ESRD was defined as the initiation of dialysis or eGFR $<15 \mathrm{ml} / \mathrm{min} / 1.73 \mathrm{~m}^{2}$. The progressive group was defined as those with the occurrence of the endpoint events, and the stable group was patients whose renal function was still maintained in stage 4 CKD. For all endpoints, the follow-up data were available until April 1, 2020 , or from the day of renal biopsy to the occurrence of either ESRD or death. No patients underwent kidney transplantation during the follow-up.

\section{Renal Biopsy and Pathologic Classification}

The indications of renal biopsy were as follows: abruptly decreased serum creatinine or persistent albuminuria, especially with rapidly progressive glomerular nephropathy, or sudden onset of marked hematuria. Therefore, the renal biopsy subjects in this study were DN patients with atypical clinical manifestations.

All the renal biopsy specimens were preserved in the China-Japan Friendship Hospital. 
For each biopsy specimen, light microscopy, immunofluorescence, and electron microscopy were routinely performed. Sections for light microscopy were stained with periodic acidSchiff, hematoxylin-eosin (H\&E), Masson trichrome, and periodic acid methenamine silver. Immunofluorescence staining was performed using antibodies against immunoglobulin (IgG, IgM, IgA), C3, C1q, and kappa and lambda light chains on frozen tissue. Transmission electron microscopy was used to detect renal pathologic alterations.

All patients were categorized based on the pathologic classification, and the histologic scores were determined based on the Renal Pathology Society's criteria [12]. The glomerular classifications were as follows: class I, mild or nonspecific light microscopy changes and electron microscopy-proven glomerular basement membrane thickening; class IIa, mild mesangial expansion; class IIb, severe mesangial expansion; class III, nodular sclerosis (KimmelstielWilson lesion); class IV, global glomerular sclerosis in $>50 \%$ of glomeruli. All the specimens were scored by two renal pathologists who were blinded to the study.

\section{Immunohistochemistry of Angiotensin II Type 1 Receptor}

Paraffin-embedded tissues were sectioned at 3 $\mu \mathrm{m}$ thickness. After dewaxing and hydration treatment, antigen recovery was performed using the high-pressure method. The sections were blocked with 3\% peroxide-methanol at room temperature for endogenous peroxidase ablation and then incubated with goat serum at room temperature for $30 \mathrm{~min}$. The antibody used in this study was the anti-AT1R antibody (Abcam, ab124505). After immunostaining, all sections were counterstained with hematoxylin.

Immunohistochemical images were acquired using a microscope at $\times 400$ magnification (Nikon, Tokyo, Japan) and an integrated digital camera system (Nikon). The expression of AT1R was semi-quantified using Image-Pro Plus software 6.0 (Media Cybernetics, Bethesda, MD) in ten consecutive fields (original magnification $\times 200$ ).

\section{Statistical Analyses}

Continuous data were regarded as nonparametric and were presented as median and interquartile range (IQR). Differences were analyzed using the Mann-Whitney $U$ nonparametric test. Categorical variables were reported as percentages and analyzed using the chi-square or Fisher's exact test. Renal outcome was evaluated using Kaplan-Meier survival analysis. Cox proportional hazard models were conducted to estimate the hazard ratio (HR) and 95\% confidence interval (CI) for data associated with stage $4 \mathrm{CKD}$ patients with $\mathrm{DN}$. The baseline parameters were assessed first performing univariate log-rank tests, and those with $p$ values $<0.10$ and the indicators that might be meaningful in clinical treatment were incorporated into the final multivariable Cox proportional hazards regression model [13]. Statistical analysis was performed using SPSS software (version 20; IBM Corp, Armonk, NY). We used the G.power software 3.1.9.2 (http://www. gpower.hhu.de/) to calculate the statistical power value [14]. Two-sided $p$ values $<0.05$ were considered to indicate statistical significance.

\section{RESULTS}

\section{Baseline Clinical and Pathologic Characteristics}

Forty-six patients with a pathologic diagnosis of DN met the inclusion criteria and were enrolled in this study. In brief, the number of male patients was $31(67.4 \%)$, and the median age was 54.0 (IQR 47.0-60.0) years. Patients in the progressive group were overweight (BMI $26.1 \mathrm{~kg} / \mathrm{m}^{2}$ ) with a slightly higher than stable group (BMI $25.6 \mathrm{~kg} / \mathrm{m}^{2}$ ). With the progression of advanced $\mathrm{DN}$, the hemoglobin value of the progressive group was significantly lower than that of the stable group $(95.0 \mathrm{~g} / \mathrm{l}$ vs. $109.0 \mathrm{~g} / \mathrm{l}, p=0.046)$. The same condition was also applicable for the management of triglycerides $(p=0.029)$. Although the duration of diabetes in the progressive group was longer than that in the stable group, there was no 
statistical difference between the two groups. Conservative treatments, such as use of ARB therapy, ESA therapy, control glucose, and Rheum officinale therapy, were not statistically different between the two groups. Only 34.8\% of patients reached the target blood pressure. Eighteen patients continued use of ARB therapy (2 losartan, 2 telmisartan, 7 valsartan, 6 irbesartan, and 1 olmesartan).

The pathologic classification was as follows: there were no cases of class IIa, 12 cases of class IIb, 27 cases of class III, and 7 cases of class IV. The baseline clinical, laboratory, and pathologic characteristics of the cohort are summarized in Tables 1 and 2.

\section{Follow-Up and Renal Outcome}

The survival curve for the condition of using ARB therapy is shown in Fig. 2a. In the 'use of ARB' group, median survival duration was 25.3 (95\% CI 19.7-30.9) months and in the 'no use

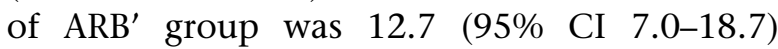
months. The overall median survival duration was 17.3 (95\% CI 7.4-27.3) months. The 1-, 2-, and 3-year renal survival rates were $58.9 \%$, $38.8 \%$, and $17.2 \%$, respectively. Primary endpoint events occurred in 29 individuals $(63.0 \%)$ during follow-up, including 24 who developed to ESRD and 5 who died before progression to ESRD. Two individuals died of heart failure, one of respiratory failure, and the other two individuals of sepsis. Results of the comparison between the main clinical parameters at baseline and during the follow-up (or at the final assessment) are shown in Table 3.

\section{Possible Related Factors to Renal Outcome}

The evaluated baseline clinical characteristic parameter of triglyceride levels was associated with renal outcome in the univariate Cox analyses at $p<0.05$. The multivariate Cox analysis showed that neither of the possible clinicopathologic factors had a significant effect on slowing the progression of $\mathrm{DN}$ duration (Table 4). Conventional therapies, such as use of ARB, glycated hemoglobin (HbA1c) level $<7 \%$, blood pressure $<130 / 80 \mathrm{mmHg}$, or use of
Rheum officinale, were also not statistically different between the stable and progressive groups.

\section{AT1R Expression}

The correlation between renal function and the expression level of AT1R is shown in Fig. 2b, c. Comparing between the stable group and the progressive group, renal expression levels of AT1R had no significant difference in the glomeruli, renal tubules, and vessels $(p>0.05)$. We also assessed the effect of ARB on intrarenal AT1R expression (18 patients with ARB, 28 patients without ARB therapy). There was no significantly differences in AT1R expression levels.

\section{DISCUSSION}

To our knowledge, our study was the first to conduct a follow-up on patients with stage 4 CKD who were also pathologically diagnosed with DN. Forty-six patients were involved in this study, and follow-up time was 11.4 (IQR 8.4-23.7) months; the median survival time was 17.3 months. We found none of the clinicopathologic data, including pathologic class of $\mathrm{DN}$, were statistically independent prognostic factors for renal survival. Conventional therapies, including the use of ARB, a level of glycated hemoglobin (HbA1c) $<7 \%$, blood pressure $<130 / 80 \mathrm{mmHg}$, or use of Rheum officinale, were also not statistically different between the stable and progressive groups.

As is well known, the clinicopathologic data on progression from stage 4 CKD to ESRD are inadequate for individuals with biopsy-proven DN. By analyzing the immunohistochemical results of AT1R expression in the two groups, we found there were no significant differences in the expression of AT1R in the glomeruli, renal tubules, and vessels in DN patients in stage 4 CKD.

We have included brief data on renal survival in DN patients with stage 4 CKD. AT1R, as a component of the RAS, plays a role in inappropriate activation in CKD progression. Unlike in animal models $[15,16]$, reports on human renal tissue have rarely been made. Research 
Table 1 Clinical characteristics of stage 4 CKD patients with diabetic nephropathy at the time of renal biopsy

\begin{tabular}{|c|c|c|c|c|}
\hline \multirow[t]{2}{*}{ Clinical parameters } & \multirow[t]{2}{*}{ Total } & \multicolumn{3}{|l|}{ Cohort $(n=46)$} \\
\hline & & Stable $(n=17)$ & Progressive $(n=29)$ & $p$ \\
\hline Sex, male (\%) & $31(67.4)$ & $14(82.4)$ & $17(58.6)$ & 0.097 \\
\hline Age (years) & $54.0(47.0-60.0)$ & $53.0(43.0-62.5)$ & $54.0(47.5-59.5)$ & 0.838 \\
\hline BMI $\left(\mathrm{kg} / \mathrm{m}^{2}\right)$ & $26.1 \pm 3.4$ & $25.6(24.2-29.3)$ & $26.1(22.9-27.6)$ & 0.829 \\
\hline Diabetes duration (years) & & & & 0.748 \\
\hline$<5$ & 13 & 4 & 9 & \\
\hline $5-<10$ & 12 & 5 & 7 & \\
\hline$\geq 10$ & 21 & 8 & 13 & \\
\hline 24 h-UPE (g/day) & $6.2(3.1-8.1)$ & $5.9(2.8-8.7)$ & $6.8(4.0-8.1)$ & 0.580 \\
\hline Serum creatinine $(\mu \mathrm{mol} / \mathrm{l})$ & $251.0(222.4-294.39)$ & $250.0(222.9-295.1)$ & $254.0(218.5-295.5)$ & 0.838 \\
\hline $\mathrm{eGFR}\left(\mathrm{ml} / \mathrm{min} / 1.73 \mathrm{~m}^{2}\right)$ & $22.3(19.0-26.9)$ & $22.1(19.2-26.8)$ & $23.3(18.7-27.6)$ & 0.946 \\
\hline Serum albumin $(\mathrm{g} / \mathrm{l})$ & $33.0(28.9-37.0)$ & $33.0(28.5-38.7)$ & $32.5(29.3-35.8)$ & 0.497 \\
\hline $\mathrm{FBG}(\mathrm{mmol} / \mathrm{l})$ & $6.0(5.0-8.0)$ & $7.0(5.0-8.5)$ & $6.0(5.0-8.0)$ & 0.669 \\
\hline HbAlc $<7 \%, n(\%)$ & $20(43.5)$ & $7(35.0)$ & $13(65.0)$ & 0.809 \\
\hline Hemoglobin $(\mathrm{g} / \mathrm{l})$ & $100.5(90.0-110.0)$ & $109.0(93.0-111.0)$ & $95.0(88.0-105.5)$ & 0.046 \\
\hline Cholesterol (mmol/l) & $5.5(4.4-6.8)$ & $5.1(4.7-6.4)$ & $5.9(4.3-7.6)$ & 0.746 \\
\hline Triglyceride $(\mathrm{mmol} / \mathrm{l})$ & $2.0(1.1-3.0)$ & $3.0(1.8-4.8)$ & $2.0(1.1-2.8)$ & 0.029 \\
\hline Potassium $(\mathrm{mmol} / \mathrm{l})$ & $4.7(4.1-5.1)$ & $4.4(3.8-4.8)$ & $4.8(4.4-5.2)$ & 0.034 \\
\hline Phosphate (mmol/l) & $1.4(1.2-1.5)$ & $1.28(1.1-1.4)$ & $1.4(1.3-1.6)$ & 0.003 \\
\hline $\mathrm{BNP}(\mathrm{pg} / \mathrm{ml})$ & $174.3(95.0-174.3)$ & $116.4(42.7-174.3)$ & $174.3(120.2-174.3)$ & 0.089 \\
\hline Parathyroid hormone $(\mathrm{pg} / \mathrm{ml})$ & $97.1(65.0-119.0)$ & $97.4(74.8-128.0)$ & $74.9(54.3-112.7)$ & 0.190 \\
\hline $\mathrm{SBP}(\mathrm{mmHg})$ & $147.0(129.5-160.5)$ & $145.0(131.5-157.5)$ & $153.0(129.0-169.5)$ & 0.393 \\
\hline $\mathrm{DBP}(\mathrm{mmHg})$ & $80.0(72.8-86.0)$ & $76.0(72.0-85.5)$ & $80.0(74.5-86.5)$ & 0.362 \\
\hline Use of ARB therapy, $n$ (\%) & $18(39.1)$ & $9(52.9)$ & $8(27.6)$ & 0.142 \\
\hline Antihypertensive therapy, $n$ (\%) & $46(100 \%)$ & $17(100 \%)$ & $29(100 \%)$ & - \\
\hline Target of $\mathrm{BP}^{\mathrm{a}}, n(\%)$ & $16(34.8)$ & $7(41.2)$ & $9(31.0)$ & 0.486 \\
\hline $\mathrm{ESA}, n(\%)$ & $24(52.2)$ & $7(41.2)$ & $17(58.6)$ & 0.253 \\
\hline Hypoglycemic therapy & & & & 0.057 \\
\hline OHA therapy & $13(28.3)$ & $2(11.8)$ & $11(37.9)$ & \\
\hline Insulin therapy & $33(71.7)$ & $15(88.2)$ & $18(62.1)$ & \\
\hline
\end{tabular}


Table 1 continued

\begin{tabular}{lllll}
\hline Clinical parameters & Total & Cohort $(\boldsymbol{n}=4 \mathbf{4 6})$ & & \\
\cline { 3 - 5 } & & Stable $(\boldsymbol{n}=\mathbf{1 7})$ & Progressive $(\boldsymbol{n}=\mathbf{2 9})$ & $\boldsymbol{p}$ \\
\hline Use of Rheum officinale therapy & $32(69.6)$ & $11(64.7)$ & $21(72.4)$ & 0.583 \\
History of CVEs & $16(34.8)$ & $4(23.5)$ & $12(41.4)$ & 0.220 \\
\hline
\end{tabular}

24 h-UPE 24-h urinary proteinuria excretion, $e G F R$ estimated glomerular filtration rate, $F B G$ fasting blood glucose, $B N P$ brain natriuretic peptide, $S B P$ systolic blood pressure, $D B P$ diastolic blood pressure, $A R B$ angiotensin II type 1 receptor blocker, ESA erythropoietin-stimulating agent, $C V E$ cardiovascular events, OHA oral hypoglycemic agent, Insulin therapy treatment with insulin including basal-supported oral therapy, $C K D$ chronic kidney disease

${ }^{a}$ BP target was $<130 / 80 \mathrm{mmHg}$

Table 2 Pathologic characteristics of stage $4 \mathrm{CKD}$ patients with DN

\begin{tabular}{llll}
\hline $\begin{array}{l}\text { Pathologic } \\
\text { stage }\end{array}$ & Stable $(\boldsymbol{n}=17)$ & $\begin{array}{l}\text { Progressive } \\
(\boldsymbol{n}=29)\end{array}$ & $p$ \\
\hline $\begin{array}{l}\text { RPS DN } \\
\text { class }^{\mathrm{a}}\end{array}$ & & & 0.046 \\
IIa & 0 & 0 & \\
IIb & 7 & 5 & \\
III & 6 & 21 & \\
IV & 4 & 3 & \\
\hline
\end{tabular}

CKD chronic kidney disease, RPS Renal Pathology Society, $D N$ diabetic nephropathy

a Scores were defined by the RPS Diabetic Nephropathy Classification ${ }^{[[18]]}$

involving 37 patients with renal biopsy showed mRNA levels of AT1R were significantly decreased in DN patients $(p<0.001)$ [17]. Previous research demonstrated that tubulointerstitial AT1R expression was significantly stronger in class IIb, and the expression was higher than that in class III + IV [18]. However, regrettably, no follow-up information or RAS inhibitor treatment was provided. By comparing the immunohistochemistry of two groups, our study found that AT1R expression levels rarely had a significant effect on the progression of DN in stage 4 CKD to ESRD. This factor may be related to the mechanism of AT1R function.
Studies have shown that inhibiting AT1R could reduce the production of fibrotic cytokines and accumulation of ECM and change the activity of nephrin to alter the structure and function of podocytes. However, in advanced DN, especially in stage $4 \mathrm{CKD}$ patients with $\mathrm{DN}$, the structure of the nephrin is severely altered, which may cause a decline in AT1R expression.

Meanwhile, information on ARB treatment and the prognosis of advanced DN in stage 4 CKD was collected, and the use of ARB drugs was of little significance in delaying the disease progression; however, several clinical trials have proven ARBS to relief macroalbuminuria by blocking the RAAS system, delaying the progression of DN and reducing the incidence of ESRD [19]. These studies focused on the initial stage of diabetes instead of the continuity of using ARB in advanced DN. Moreover, two large, prospective, randomized, double-blind clinical trials reported that the effects of AT1R antagonists, losartan and irbesartan, reduced the incidence of ESRD with no difference in blood pressure and mortality [20]. The possible reason was the renal functions of the individuals were quite different and were not in the same stage. In our study, the renal function of patients was worse, and the pathologic damage was more serious. At this stage, the effect of ACEI or ARB treatment is not optimal.

The goal blood pressure of CKD is $<130 / 80 \mathrm{mmHg}$, and few patients in our study achieved the goal. Simply controlling the BP at lower than the target value was not an 

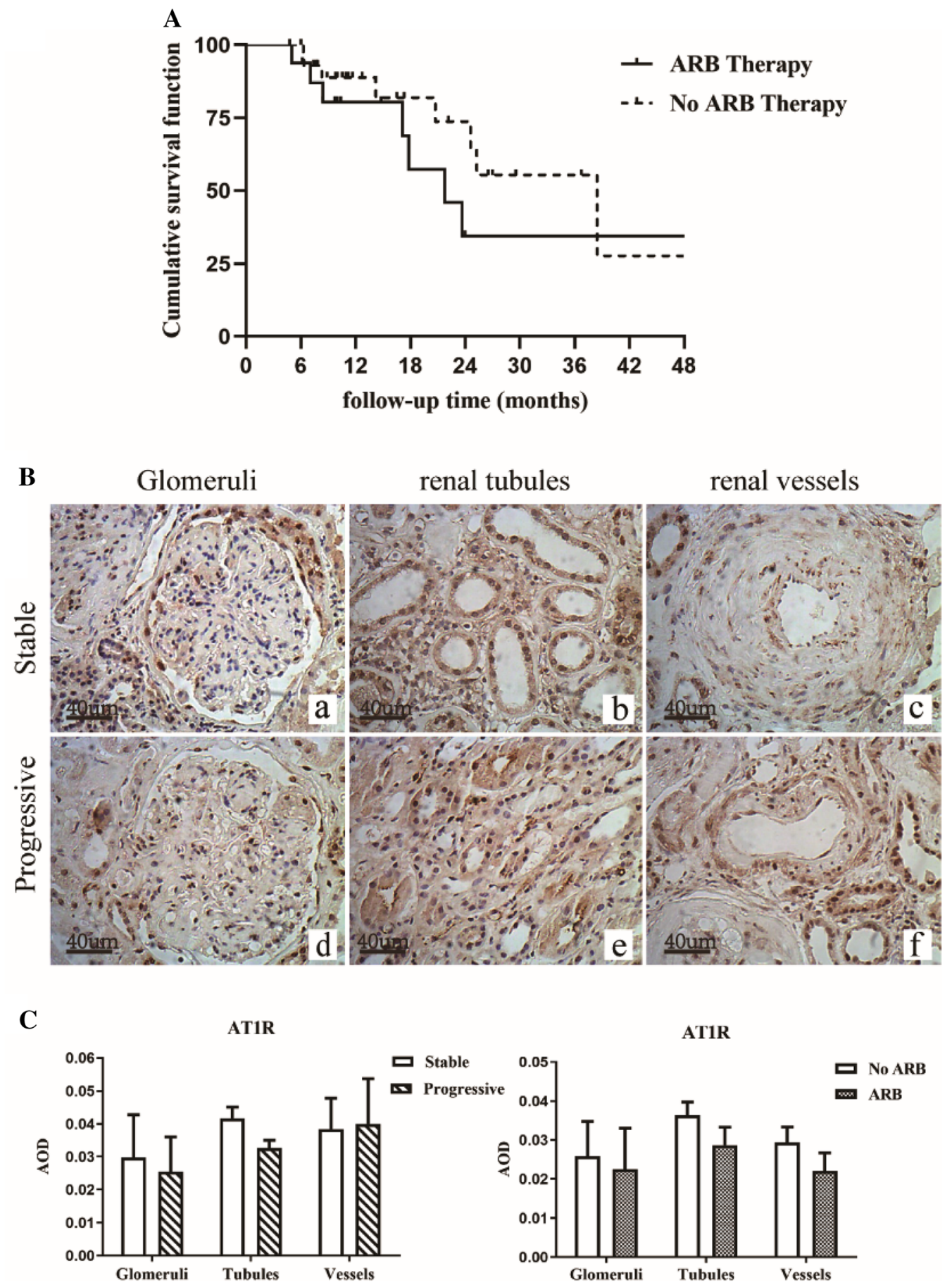

Fig. 2 a Renal survival in stage 4 chronic kidney disease (CKD) patients with diabetic nephropathy. b Immunohistochemical staining for angiotensin II type 1 receptor (AT1R) expression in the glomeruli, renal tubules, and renal vessels of stage $4 \mathrm{CKD}$ patients with diabetic

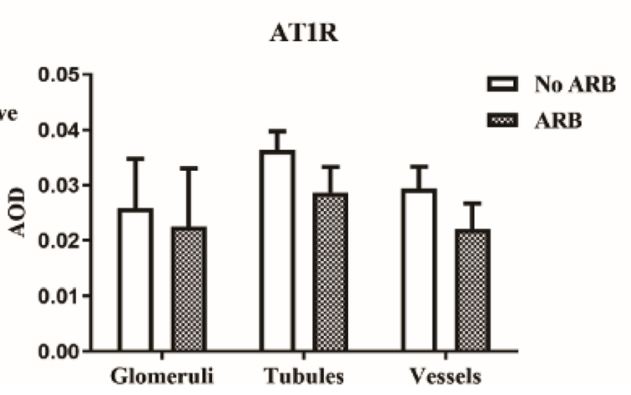

nephropathy $(\mathrm{DN})$ in the stable group and the progressive group (original magnification $\times 200$ ). $\mathbf{c}$ Expression levels of AT1R in the glomeruli, renal tubules, and renal vessels of the stable group and the progressive group. $A O D$ average optical density. ${ }^{*} p<0.05$ versus stable group 
Table 3 Comparison of main clinical parameters between baseline and follow-up in stage 4 CKD patients with diabetic nephropathy

\begin{tabular}{lllr}
\hline Clinical parameters & Baseline & During follow-up or at final follow-up & $\boldsymbol{p}$ value \\
\hline Serum creatinine $^{\mathrm{a}}(\mu \mathrm{mol} / \mathrm{l})$ & $251.0(222.4-294.39)$ & $540.4(304.7,695.0)$ & $<0.001$ \\
ARB therapy (\%) & 39.1 & 23.9 & 0.116 \\
OHA therapy (\%) & 28.3 & 23.9 & 0.635 \\
Insulin therapy (\%) & 71.7 & 76.1 & 0.635 \\
ESA (\%) & 52.2 & 63.0 & 0.291 \\
Outcome (\%) & & $63.0(n=29)$ & \\
Renal event & & $52.2(n=24)$ & \\
Death & & $10.9(n=5)$ & \\
\hline
\end{tabular}

Use of an ARB during the follow-up was defined as treatment with the relevant drug for $>3$ months

$C K D$ chronic kidney disease, $A R B$ angiotensin II type 1 receptor blocker, $O H A$ oral hypoglycemic agent, Insulin therapy treatment with insulin including basal-supported oral therapy, ESA erythropoietin-stimulating agent, Renal event commencement of dialysis because of ESRD

${ }^{a}$ Median (interquartile range)

important contributor. It has been reported that the protection of ARB is independent of the BPlowering effect on DN progression [21].

As a traditional Chinese medicine treatment, the Chinese patented medicine Rheum (CPMRheum) is widely used in patients with CKD. Rheum officinale may have a positive effect on creatinine values (mean $87.49 \mu \mathrm{mol} / \mathrm{l}$ ) but no evidence is available on the all-cause mortality or cost of therapy for CKD. Another metaanalysis study [6] showed CPM-Rheum combined with ARB or ACEI could lower creatinine levels and attenuated the impairment of kidney function. Our study demonstrated CPM-Rheum had little effect on delaying the progression to ESRD in DN patients with stage 4 CKD. Regrettably, there is currently no high-quality evidence to support any consensus recommendations for its use.

Our research study had several strengths. First, to our knowledge, this study is the first to investigate DN in stage 4 CKD. Second, the DN patients enrolled in this study were diagnosed by renal biopsy. Lastly, we assessed the time to the development of ESRD based on the followup data of stage $4 \mathrm{CKD}$ patients with $\mathrm{DN}$, and this information may be used as a reference for future clinical evaluations.

Despite these strengths, our study also had several limitations. First, the sample size was small because few diabetic patients with kidney disease underwent renal biopsy, especially in stage $4 \mathrm{CKD}$. The biopsied DN patients in the present study were clinically atypical. Therefore, the results may not be applicable to all DN patients with stage $4 \mathrm{CKD}$. Hence, our findings will require confirmation in a multicenter study. Second, this study had a retrospective design and failed to retain blood and urine samples for further research and analysis. Third, the renal tissue specimens obtained from the renal biopsy did not have enough volume to extract protein to carry out other more diversified experiments.

\section{CONCLUSION}

In conclusion, this study showed that most patients $(60.3 \%)$ rapidly progressed to ESRD or died (10.9\%) after being diagnosed with stage 4 CKD with DN. In such patients, adding ARB 
Table 4 Univariate associations between candidate predictors and survival and multivariate Cox regression analysis of stage 4 CKD patients with DN

\begin{tabular}{|c|c|c|c|c|}
\hline \multirow[t]{2}{*}{ Variables } & \multicolumn{2}{|l|}{ Univariate analysis } & \multicolumn{2}{|c|}{ Multivariate analysis } \\
\hline & HR (95\%CI) & $p$ & HR (95\%CI) & $p$ \\
\hline 24 h-UPE (g/day) & $1.04(0.95-1.14)$ & 0.37 & & \\
\hline Serum albumin $(g / l)$ & $0.94(0.87-1.02)$ & 0.11 & & \\
\hline Serum creatinine $(\mu \mathrm{mol} / \mathrm{l})$ & $0.99(0.98-1.00)$ & 0.36 & & \\
\hline $\operatorname{eGFR}\left(\mathrm{ml} / \mathrm{min} / 1.73 \mathrm{~m}^{2}\right)$ & $1.04(0.96-1.13)$ & 0.37 & & \\
\hline Triglyceride $(\mathrm{mmol} / \mathrm{l})$ & $0.66(0.44-0.99)$ & 0.04 & $0.70(0.42-1.18)$ & 0.18 \\
\hline Cholesterol (mmol/l) & $1.13(0.92-1.38)$ & 0.23 & & \\
\hline FBG $(\mathrm{mmol} / \mathrm{l})$ & $0.98(0.85-1.12)$ & 0.77 & & \\
\hline $\operatorname{HbAlc}(<7 \%)$ & $1.13(0.93-1.37)$ & 0.23 & $1.10(0.86-1.40)$ & 0.46 \\
\hline Hemoglobin $(\mathrm{g} / \mathrm{l})$ & $1.00(0.98-1.02)$ & 0.60 & & \\
\hline Potassium (mmol/l) & $1.52(0.95-2.42)$ & 0.08 & & \\
\hline Phosphate $(\mathrm{mmol} / \mathrm{l})$ & $4.18(0.90-19.31)$ & 0.07 & & \\
\hline $\mathrm{BNP}(\mathrm{pg} / \mathrm{ml})$ & $1.00(0.99-1.01)$ & 0.94 & & \\
\hline Parathyroid hormone $(\mathrm{pg} / \mathrm{ml})$ & $0.99(0.99-1.01)$ & 0.79 & & \\
\hline $\mathrm{SBP}(\mathrm{mmHg})$ & $1.00(0.98-1.02)$ & 0.75 & & \\
\hline $\mathrm{DBP}(\mathrm{mmHg})$ & $1.01(0.98-1.04)$ & 0.38 & & \\
\hline Target of $\mathrm{BP}^{\dagger}, n(\%)$ & $1.30(0.58-2.91)$ & 0.52 & $1.06(0.37-3.03)$ & 0.92 \\
\hline RPS DN class & $1.47(0.72-2.79)$ & 0.26 & $0.85(0.34-2.14)$ & 0.73 \\
\hline Use of ARB therapy & $0.66(0.30-1.49)$ & 0.32 & $0.92(0.41-2.08)$ & 0.85 \\
\hline Use of Rheum offcinale therapy & $1.04(0.45-2.41)$ & 0.92 & & \\
\hline
\end{tabular}

24 h-UPE, 24-h urinary proteinuria excretion; eGFR, estimated glomerular filtration rate; FBG, fasting blood glucose; BNP, brain natriuretic peptide; RPS, Renal Pathology Society; DN, diabetic nephropathy

$\dagger$ BP target $<$ than $130 / 80 \mathrm{mmHg}$

drugs and CPM-Rheum, or targeting $\mathrm{BP}<130 /$ $80 \mathrm{mmHg}$ and a reasonable HbA1c concentration, could barely delay the progression of advanced DN to ESRD. Therefore, we should intervene in a timely manner in the management of early stage $\mathrm{DN}$ and slow the progression of the disease. For DN patients who have entered stage $4 \mathrm{CKD}$, interventions should focus on both the prevention of progression of diabetic nephropathy to reduce the mortality rate and allow future resource planning.

\section{ACKNOWLEDGEMENTS}

We thank the participants of the study.

Funding. This study and the Rapid Service Fee were supported by grants from the Science 
and Technology Project of Beijing (D171100002817003), China Health Promotion Foundation (DKD-MBD project, 2018-2022), Beijing Natural Science Foundation (7202179).

Additional Assistance. The authors thank the Department of Nephrology, China-Japan Friends Hospital, for providing the technical assistance.

Authorship. All named authors meet the International Committee of Medical Journal. Editors (ICMJE) criteria for authorship for this article, take responsibility for the integrity of the work as a whole, and have given their approval for this version to be published.

Authorship Contributions. Wenge Li and Shimin Jiang were responsible for the conception and design of the study. Tianyu Yu was responsible for data acquisition, data analysis, and drafting. Shimin Jiang and Jinying Fang were the chief investigators and were also responsible for the data analysis. Yue Yang, Guming Zou, Hongmei Gao, and Li Zhuo were responsible for the research data analysis. All authors contributed to the writing of the final manuscript.

Disclosures. Tianyu Yu, Shimin Jiang, Yue Yang, Jinying Fang, Guming Zou, Hongmei Gao, Li Zhuo, and Wenge Li have nothing to disclose.

Compliance with Ethics Guidelines. The study was approved by the institutional review boards of China-Japan Friendship Hospital (2018-43-K32). All procedures were performed in accordance with the Helsinki Declaration of 1964 and its later amendments and were in agreement with national regulations. All patients provided written informed consent to reuse their clinical data for research purposes.

Data Availability. The datasets generated during and/or analyzed during the current study are available from the corresponding author on reasonable request.
Open Access. This article is licensed under a Creative Commons Attribution-NonCommercial 4.0 International License, which permits any non-commercial use, sharing, adaptation, distribution and reproduction in any medium or format, as long as you give appropriate credit to the original author(s) and the source, provide a link to the Creative Commons licence, and indicate if changes were made. The images or other third party material in this article are included in the article's Creative Commons licence, unless indicated otherwise in a credit line to the material. If material is not included in the article's Creative Commons licence and your intended use is not permitted by statutory regulation or exceeds the permitted use, you will need to obtain permission directly from the copyright holder. To view a copy of this licence, visit http://creativecommons.org/licenses/by$\mathrm{nc} / 4.0 /$.

\section{REFERENCES}

1. Ruiz-Ortega M, Rayego-Mateos S, Lamas S, Ortiz A, Rodrigues D. Targeting the progression of chronic kidney disease. Nat Rev Nephrol. 2020;16(5): 269-88.

2. World Health Organization:Diabetes. https://www. who.int/en/news-room/fact-sheets/detail/diabetes.

3. de Boer IH, Rue TC, Hall YN, Heagerty PJ, Weiss NS, Himmelfarb J. Temporal trends in the prevalence of diabetic kidney disease in the United States. JAMA. 2011;305(24):2532-9.

4. International Diabetes Federation. IDF Diabetes Atlas-9th Edition. 2019, Diabetes Atlas. https:// www.diabetesatlas.org.

5. Umanath $\mathrm{K}$, Lewis JB. Update on Diabetic Nephropathy: Core Curriculum 2018. Am J Kidney Dis. 2018;71(6):884-95.

6. Yang Y, Ma YP, Zhang Z, Dai PL, Li P, Li WG. Effects of adding Rheum officinale to angiotensin-converting enzyme inhibitors or angiotensin receptor blockers on renal function in patients with chronic renal failure: a meta-analysis of randomized controlled trials. Clin Nephrol. 2018;89(6):445-54.

7. Zhang ZH, Vaziri ND, Wei F, Cheng XL, Bai X, Zhao YY. An integrated lipidomics and metabolomics reveal nephroprotective effect and biochemical 
mechanism of Rheum officinale in chronic renal failure. Sci Rep. 2016;6:22151.

8. Palmer SC, Mavridis D, Navarese E, et al. Comparative efficacy and safety of blood pressure-lowering agents in adults with diabetes and kidney disease: a network meta-analysis. Lancet. 2015;385(9982): 2047-56.

9. Inker LA, Schmid CH, Tighiouart $\mathrm{H}$, et al. Estimating glomerular filtration rate from serum creatinine and cystatin C. N Engl J Med. 2012;367(1):20-9.

10. KDOQI. KDOQI clinical practice guidelines and clinical practice recommendations for diabetes and chronic kidney disease. Am J Kidney Dis. 2007;49(2 Suppl 2):S12-154.

11. ADA.6. Glycemic targets: standards of medical care in diabetes-2019. Diabetes Care. 2019;42(Suppl 1): S61-70.

12. Tervaert TW, Mooyaart AL, Amann K, et al. Pathologic classification of diabetic nephropathy. J Am Soc Nephrol. 2010;21(4):556-63.

13. Benítez-Parejo N, Rodríguez del Áuila MM, PérezVicente S. Survival analysis and Cox regression. Allergol Immunopathol (Madr). 2011;39(6):362-73.

14. Faul F, Erdfelder E, Lang AG, Buchner A. G*Power 3: a flexible statistical power analysis program for the social, behavioral, and biomedical sciences. Behav Res Methods. 2007;39(2):175-91.

15. Hu J, Tiwari S, Riazi S, Hu X, Wang X, Ecelbarger CM. Regulation of angiotensin II type I receptor
(AT1R) protein levels in the obese Zucker rat kidney and urine. Clin Exp Hypertens. 2009;31(1):49-63.

16. Xu ZG, Miao LN, Cui YC, Jia Y, Yuan H, Wu M. Angiotensin II type 1 receptor expression is increased via 12-lipoxygenase in high glucosestimulated glomerular cells and type 2 diabetic glomeruli. Nephrol Dial Transplant. 2009;24(6): 1744-52.

17. Wagner J, Gehlen F, Ciechanowicz A, Ritz E. Angiotensin II receptor type 1 gene expression in human glomerulonephritis and diabetes mellitus. J Am Soc Nephrol. 1999;10(3):545-51.

18. Ma YP, Yang Y, Jiang SM, et al. Angiotensin II type 1 receptor blockers favorably affect renal angiotensin II and MAS receptor expression in patients with diabetic nephropathy. J Renin Angiotensin Aldosterone Syst. 2020;21(2):1470320320919607.

19. Haller H, Viberti GC, Mimran A, et al. Preventing microalbuminuria in patients with diabetes: rationale and design of the Randomised Olmesartan and Diabetes Microalbuminuria Prevention (ROADMAP) study. J Hypertens. 2006;24(2):403-8.

20. Brenner BM, Cooper ME, de Zeeuw D, et al. Effects of losartan on renal and cardiovascular outcomes in patients with type 2 diabetes and nephropathy. N Engl J Med. 2001;345(12):861-9.

21. Zhu Y, Li ZL, Ding A, et al. Olmesartan Medoxomil, An Angiotensin II-Receptor Blocker, Ameliorates Renal Injury In db/db Mice. Drug Des Devel Ther. 2019;13:3657-67. 